\title{
EINLADUNG ZUR JAHRESTAGUNG DER ARBEITSGEMEIN- SCHAFT FÜR MEDIZINISCHES BIBLIOTHEKSWESEN (AGMB) AN DIE VETERINÄRMEDIZINISCHE UNIVERSITÄT WIEN VON 25. BIS 27. SEPTEMBER 2017
}

\section{von David Frank}

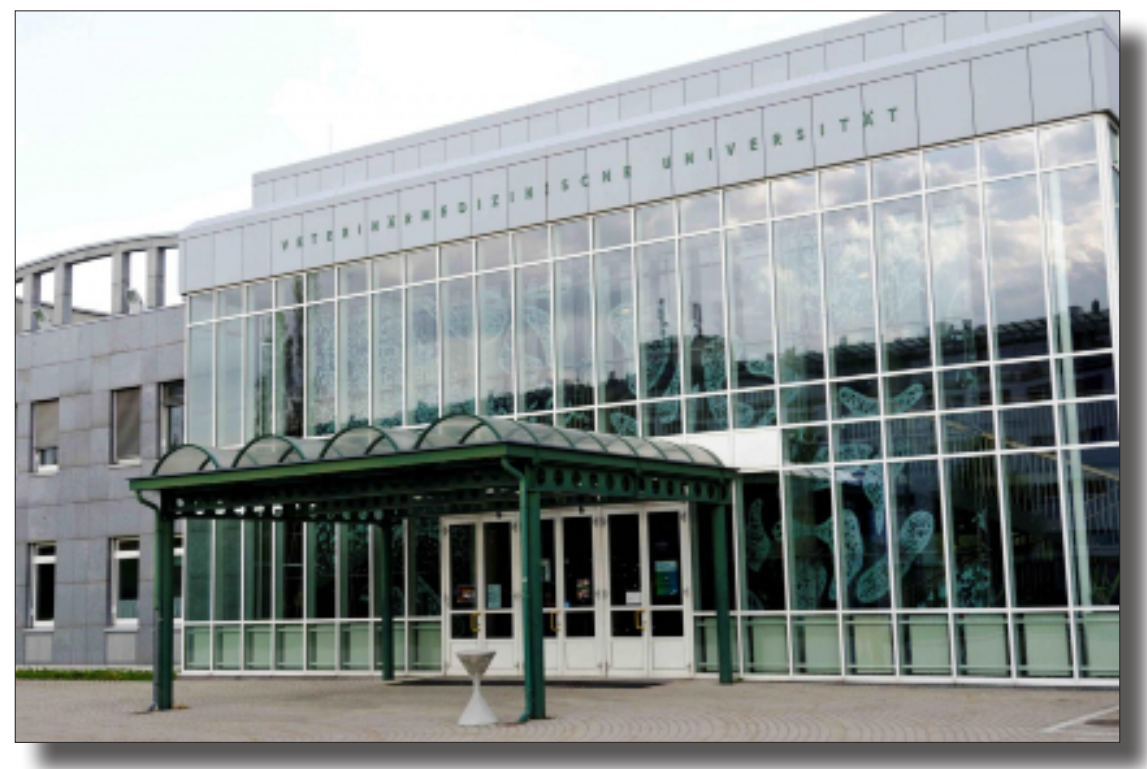

Abb. 1: Festsaalgebäude der Vetmeduni Vienna, in dem die AGMB-Jahrestagung 2017 stattfinden wird. (Foto: Michael Bernkopf/Vetmeduni Vienna)

Die Universitätsbibliothek der Veterinärmedizinischen Universität Wien ist in diesem Jahr erstmalig Ausrichterin der größten medizinbibliothekarischen Tagung im deutschen Sprachraum. Unter dem Motto „Medizinbibliotheken: inter:disziplinär - inter:national - inter:aktiv“ werden etwa 120 bis 150 Teilnehmerlnnen, die überwiegend aus Deutschland anreisen, erwartet. Bedingt durch den Veranstaltungsort Wien erwarten die Veranstalter in diesem Jahr überproportional viele Bibliothekarlnnen aus Österreich.

In vielerlei Hinsicht verspricht diese Tagung eine besondere zu werden, denn:

- es ist die erste AGMB-Jahrestagung an einer veterinärmedizinischen Einrichtung. Die Universitätsbibliothek der Veterinärmedizinischen 
Universität Wien verbindet damit das Ziel die Sichtbarkeit der Veterinärmedizin innerhalb der Medizin zu erhöhen.

- Noch nie wurde eine AGMB-Jahrestagung im deutschen Sprachraum geografisch so weit im Osten veranstaltet. Aus diesem Grund möchten die Veranstalterlnnen auch den Blick über den Tellerrand fördern und Einrichtungen aus den angrenzenden Ländern ermutigen als Vortragende bzw. Besucherlnnen an der Tagung teilzunehmen.

- es ist die größte Veranstaltung, die die Universitätsbibliothek der Veterinärmedizinischen Universität Wien bisher organisiert hat. In den letzten Jahren war die Universitätsbibliothek verstärkt Ausrichterin verschiedener bibliothekarischer Zusammenkünfte wie dem Treffen der SystembibliothekarInnen, ubifo-Sitzungen oder Veranstaltungen im Rahmen der Kooperation E-Medien Österreich.

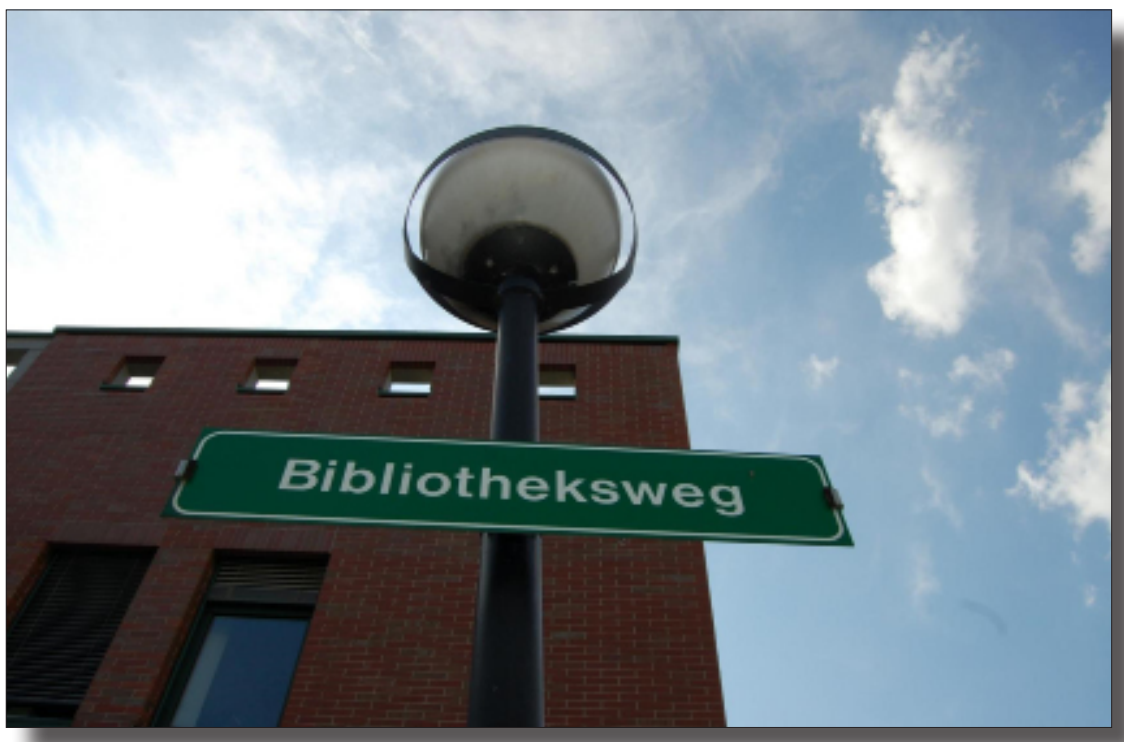

Abb. 2: Auf dem Campus der Vetmeduni Vienna (Foto: Vetmeduni Vienna)

Die Universitätsbibliothek und die AGMB freuen sich zusätzlich über eine rege Teilnahme von FachkollegInnen, die keine AGMB-Mitglieder sind und mehr über die Vereinigung erfahren möchten, in der Krankenhausbibliotheken, Informationseinrichtungen pharmazeutischer Unternehmen sowie Universitäts- und Hochschulbibliotheken vertreten sind.

Der erste Tag der Veranstaltung wird von der Tagungseröffnung dominiert, zu der hochkarätige FestrednerInnen erwartet werden. Im Anschluss 
daran finden die Treffen der beiden Arbeitskreise Medizinbibliotheken an Hochschulen sowie Krankenhausbibliotheken und die Mitgliederversammlung der AGMB statt.

Am Dienstag finden zum einen zahlreiche Vorträge und zum anderen Product Reviews durch FirmenvertreterInnen statt. Neben den klassischen Vorträgen (20 Minuten Vortrag und 10 Minuten Diskussion) gibt es Raum für Kurzvorträge, die fünf Minuten Vortrag und fünf Minuten Diskussion umfassen. Sehr positiv sind vielen Tagungsteilnehmerlnnen in den vergangenen Jahren die Treffpunkte in Erinnerung, die einen Meinungsaustausch in kleinen Gruppen, in aller Regel über ein aktuelles Thema, ermöglichen.

Wie bei zahlreichen Konferenzen üblich, darf auch eine Posterausstellung nicht fehlen. Diese steht unter dem Titel „Aus der Praxis für die Praxis".

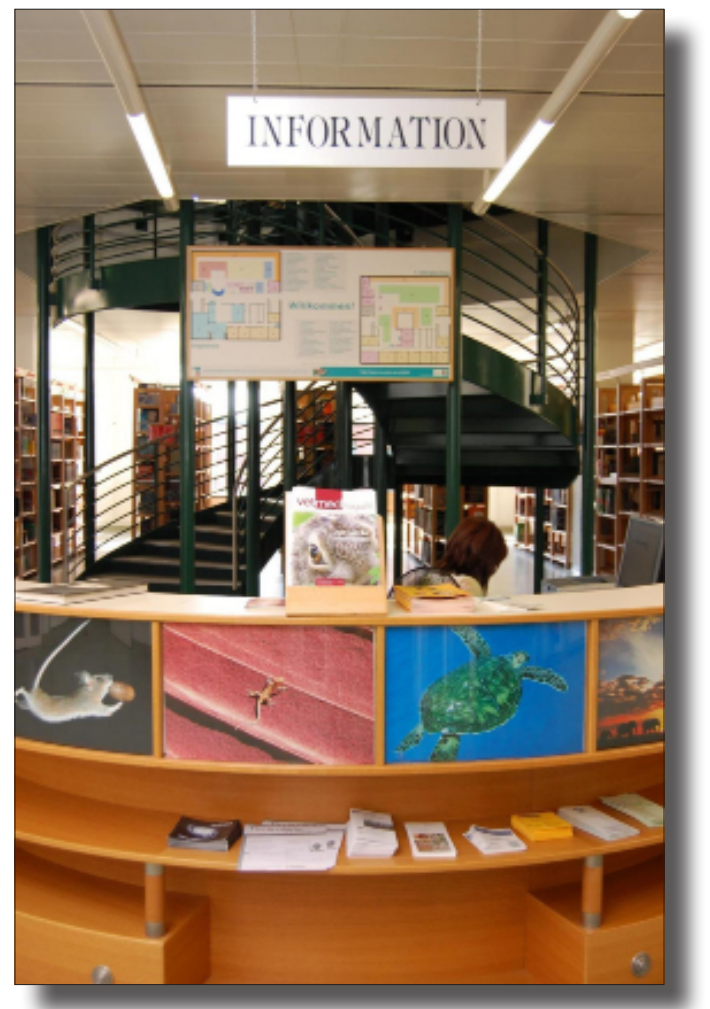

Abb. 3: Anlaufpunkt für viele Nutzerlnnen. Die Information in der Universitätsbibliothek (Foto: Vetmeduni Vienna) 
Am dritten und letzten Tag der Tagung stehen Vorträge sowie die Preisverleihung im Rahmen des Leuchtturmwettbewerbs für außergewöhnliche Projekte bzw. Leistungen von einzelnen Medizin-Bibliotheken auf dem Programm. Auch wenn es bei einer solchen Programmfülle für 3 Tage unglaublich klingen mag, aber im Rahmen der Tagung werden auch mehrstündige medizinbibliothekarische Fortbildungsveranstaltungen angeboten.

Begleitet wird die AGMB-Tagung durch eine internationale Firmenausstellung mit über 20 Ausstellern aus Europa und den USA.

Mehr als erwähnenswert ist auch das äußerst kurzweilige Rahmenprogramm, das die Veranstalterlnnen für die Teilnehmerlnnen erarbeitet haben.

Details über die Arbeitsgemeinschaft für medizinisches Bibliothekswesen (AGMB) sowie über die Jahrestagung finden sich unter http://www. agmb.de .Das Programm der Jahrestagung wird voraussichtlich ab Anfang Juni 2017 verfügbar sein.

Im Open Access-Publikationsportal German Medical Science werden die Abstracts zur Konferenz unter GMS Medizin - Bibliothek - Information (http://www.egms.de/static/de/journals/mbi/meetings.htm) veröffentlicht werden.

Als Einstimmung zur bevorstehenden Konferenz tritt sich der Arbeitskreis Österreichische Medizinbibliothekarinnen und -bibliothekare bereits am 15. Mai 2017 an der Veterinärmedizinischen Universität Wien. Dieses Treffen findet meist einmal jährlich an einer medizinischen Bibliothek statt und dient dem Erfahrungsaustausch. Die Einladung hierzu erfolgt über die Mailingliste der Vereinigung Österreichischer Bibliothekarinnen und Bibliothekare (VÖB).

Für Rückfragen zur Tagung steht der Autor gerne zur Verfügung.

\section{Dipl.-Bibliothekar (FH) David Frank Universitätsbibliothek der Veterinärmedizinischen Universität Wien \\ (Vetmeduni Vienna) Veterinärplatz 1, A-1210 Wien E-Mail: david.frank@vetmeduni.ac.at}

Dieses Werk ist lizenziert unter einer

Creative-Commons-Lizenz Namensnennung 4.0 International 